\title{
The memorial structure of organized sequences
}

\author{
NEAL F. JOHNSON \\ Ohio State University, Columbus, Ohio 43210
}

\begin{abstract}
Subjects were asked to memorize a sequence of nine consonants which were grouped into three groups of three letters each (e.g., SBJ FQL ZNG). After learning the sequence, they were presented with single letters, letter pairs, or letter triples and asked to indicate if the probe item appeared in the memorized sequence. The latency results suggest that subjects engage in a linear self-terminating memory search in which the items from one chunk are retrieved from memory as the items from the immediately preceding chunk are being scanned. When the probe consisted of more than one item, the subjects were slowed in their comparison if the letters came from different chunks (e.g., JF vs. FQ in the above illustration), and the number of letters in the probe also influenced the reaction time. Neither of those effects were obtained if all the letters in the probe came from the same chunk, and that would seem to suggest that the probe items from the same chunk were compared in parallel to the letters in the memory item, while items from different chunks were compared serially.
\end{abstract}

It has become increasingly clear that when subjects learn an organized sequence, the organization itself becomes a critical component of the learned response. In fact, if the organization of a response is altered, subjects tend to treat it as a completely new response, even when the response is otherwise unchanged (Bower \& Winzenz, 1969; Johnson, 1970; Johnson \& Migdoll, 1971). In addition, it seems that one of the primary functions of the organizational component of a response is that it serves as a generative mechanism during the process of recall. For example, it has been assumed (Johnson, 1970, 1972) that if a subject is to recall an ordered and organized sequence such as SBJ FQL ZNG (with the organization being defined by the grouping), he does so by (1) implicitly retrieving the items from a group or chunk and registering them in active working memory, (2) scanning the retrieved items to make certain that they are all known, and (3) producing the items overtly if they are all known, but completely terminating the response attempt if he is uncertain of any item.

In addition to these points, however, it is further assumed that during the scan and overt production of one chunk, the subject is free to begin the retrieval of the information from the next chunk. If these scan and retrieval processes can be done in parallel, the items from a chunk should be immediately available for processing within working memory the moment the processing of the preceding chunk is completed, and it is this mechanism which has been assumed to account for the speed and efficiency with which the

Part of the work reported herein was completed while the author was a visiting professor at the University of California, Berkeley, California. Reprint requests should be sent to Neal F. Johnson, Department of Psychology, Ohio State University, Columbus, Ohio 43210. items in organized sequences can be produced (Lashley, 1951; Johnson, 1972).

While there is general support for this model as a characterization of processing during the overt recall of an ordered and organized response sequence (Johnson, 1970, 1972), the present concern was to determine the extent to which the model also would be adequate as an account of the way the same type of sequence would be scanned when overt production was not required. The issue, then, was to explore the model's assumptions regarding the nature of the prerecall scan in a manner that is somewhat more direct than has been used in the past.

The general approach was to use Sternberg's (1969, 1975) fixed-set memory scanning task. In that situation the subjects are asked to prememorize a set of items followed by the presentation of a series of single-item displays, with the issue being the subjects' speed in ascertaining whether or not the presented item had appeared within the prememorized set (errors are kept at a minimum). It is assumed that a subject's decision is dependent upon an implicit scan of the prememorized set, and the general question examined has concerned the nature of the scanning process and the variables that influence it. In the present case, the issue is the role organization might play in directing the scanning process.

There have been a number of other studies that also have dealt with scanning processes where the to-bescanned material possessed a category or grouping structure (e.g., Crain \& De Rosa, 1974; Homa, 1973). However, these studies have tended to involve the role of organization in the process of identifying or selecting the ensemble to be scanned, rather than exploring its function in directing the scan once the appropriate ensemble has been selected. For example, Homa (1973) demonstrated that subjects can use the category 
membership of a probe item as a basis for determining which category to scan, but the scan of the category itself appeared to be serial and exhaustive, with no evidence that any within-category organization was used during the process. The present experiment, on the other hand, was more concerned with the possible role of organization in directing the scan, and that was accomplished by using an organization that did not allow the subjects to use group membership as a basis for determining what should be scanned.

Instead of the usual procedure of providing the subjects with a relatively unordered and unorganized memory set, and allowing them to structure the task in any way they choose, the present task required the subjects to memorize a chunked sequence of consonants in a specific order (e.g., SBJ FQL ZNG). In order to insure that the subjects followed the instruction, the criterion for having prememorized the set was that it could be produced in the proper order both without error and at a high speed. In addition, the chunking scheme was induced by printing the first three letters in one color, the second three in another color, and the third three in a third color. Finally, the prememorization instructions encouraged the subjects to chunk the sequence into three groups of three letters each, and they emphasized the importance of both speed and accuracy.

Regarding the general mode of the scan, the present task seems to conform to the type of situation Sternberg (1969) has described as characteristically yielding a serial self-terminating search (i.e., an emphasis on order information), and the design of the experiment was predicated on that assumption. Fortunately, it also is possible to ascertain from the data whether that boundary condition was met by examining the serial position curves. For example, if the subjects made an exhaustive scan of the whole sequence before responding, the serial position of the probe should have no influence on the reaction time, and the serial position curve would be flat. On the other hand, if they exhaustively scanned the contents of each chunk before responding, but terminated their scan if the target item appeared within the chunk, then the intrachunk serial position curves would be flat, but there would be marked increases in reaction time from the last item of one chunk to the first item of the next. Finally, if, as assumed, the entire scan is serial and self-terminating, then there would be a linear increase in the reaction time to the probe from the first item in the sequence to the last, and the effect should occur for both within-chunks and between-chunks transitions. In addition, Sternberg (1969) has reported that under these conditions the latencies should run quite high in comparison to the usual $400 \mathrm{msec}$ or so for the zero intercept. ${ }^{1}$

In terms of the specific hypothesis to be examined, if subjects do retrieve all the items from a chunk while the items from the preceding chunk are being scanned, then those items should be immediately available for scanning when the scan of the preceding chunk is completed. That is, the scan transition from the last item of one chunk to the first item in the next should be very fast and efficient, and no unusual latency effects would be expected at the interchunk transitions. On the other hand, if the scan of one chunk and the retrieval of the next cannot occur in parallel, then there should be delays at the interchunk transitions while the subject attempts to retrieve the items from the next chunk.

A second issue concerns whether organizational effects can be detected during the comparison process itself. To examine this issue, a second set of conditions was included in which the probes consisted of either two or three letters, and the subject was to determine whether the probe items occurred adjacently within the memorized sequence. The critical point of interest was to compare a condition in which the probe consisted of adjacent items from the same chunk with a condition in which the probe items were adjacent within the sequence, but came from different chunks (e.g., the last item of one chunk and the first item in the next). In both cases the appropriate response would be "yes," but the question is whether having the items come from different chunks slows the reaction time.

In that the items from a single chunk are assumed to be available at the same time, a probe that consists of items from the same chunk should result in a fast response if the comparison process is holistic or in parallel. On the other hand, if the probe items are adjacent in the sequence, but from different chunks, a sequential comparison would be required. That is, it is assumed that the items from different chunks are not available at the same time, and a partial match between the probe and the contents of one chunk would entail a subsequent comparison between the remaining parts of the probe and the contents of the next chunk. Therefore, if the comparison is done in parallel, and the items come from the same chunk, the reactions should be fast and not influenced by the size of the probe, but if the items come from different chunks, the comparison would have to be sequential and the reaction should be slow.

While this expectation is based on the assumption that the intrachunk comparison process is holistic or parallel, that assumption can be verified independently within the data. That is, if the comparison process is serial, then the size of the probe should influence the reaction time even when the items all come from the same chunk, but that should not be the case if the comparison is holistic.

To summarize, a model has been described of the way in which ordered and organized (chunked) sequences (e.g., SBJ FQL ZNG) are retrieved from memory and recalled. A critical assumption of the 
model is that subjects retrieve all the items from a chunk at the same time, and they are assumed to do so as they scan and produce the items from the immediately preceding chunk. The major implication of this model is that interchunk transitions during the scan and recall process should occur with both ease and alacrity, and that does appear to be the case.

The present issue is the extent to which that model can be used to account for the scan process in a Sternberg $(1969,1975)$ task when no overt production is required. If the same type of processing occurs in the pure scan situation, the latency at chunk boundaries should not be any longer than at within-chunks transitions. The only limitation on that expectation would occur if subjects used a strategy involving some type of exhaustive scanning, but if that occurred it would be apparent in the data in other ways (i.e., the intrachunk serial position of the probe would not influence reaction time).

The other implication of the model is that if subjects need to determine whether a multiletter probe appeared within the sequence, the reaction time should be much slower if the probe consists of items from more than one chunk than if they are all from the same chunk. A limitation on this hypothesis would occur if subjects compared the probe items with the memorized sequence in a serial manner rather than in parallel, but, again, the limitation can be detected in the data. That is, a serial comparison process also would be affected by the size of the probe, while that would not necessarily be the case if the comparison was in parallel.

\section{METHOD}

When the subjects arrived for the experiment, they were handed a $3 \times 5$ in. card that contained the sequence to be learned, and they were asked to memorize it. They also were told that their memory of the letters in the sequence would later be tested. The sequences contained nine letters, and they were printed on the card in block letters. The first three letters were printed in red, the second three letters were printed in blue, and the last three letters were printed in green, and they were all immediately adjacent to each other. Within the experiment, four different letter sequences were used (SBJFQLZNG, TBLZFJDNH, CKPMRVHWF, and NCTBXFDGR) and onequarter of the subjects learned each sequence.

The subjects were told that it would help if they would try to think of the sequence in terms of three groups of three letters each, and that the groups were color coded to help them. It usually took the subjects about $5 \mathrm{~min}$ to memorize the sequence, and then the experimenter asked them to repeat the sequence as fast as they could several times. If a subject was slow in his recitation, or he made errors, the experimenter asked him to spend more time studying the sequence.

A test stage consisted of a series of 144 displays, and each display was either a single letter, a letter bigram, or a letter trigram. In each case the subject was to indicate by pressing buttons whether the presented letter or letter sequence appeared within the sequence he had memorized.

The sequence of 144 displays was divided into six blocks of 24 displays each, which allowed the subject a short rest interval every 24 displays. Within the series, the single-, double-, and triple-letter displays occurred randomly, and the subjects did not know in advance how many letters would be in a display until it appeared. The displays lasted for $7 \mathrm{sec}$, which exceeded the duration of even the longest reaction time. The letters in the displays were printed in black and they were $1 / 2 \mathrm{in.} \mathrm{high} \mathrm{and}$ $3 / 8$ in. wide. A Scientific Prototype two-channel tachistoscope was used for presenting the displays.

For any one sequence it was possible to construct nine different single-letter displays, eight two-letter displays, and seven three-letter displays. Each of the possible displays appeared three times within the series of 144 , and on another three occasions each possible display was altered in some way, and these displays were the foils (i.e., "no" responses). For the 27 single-letter foils, the target letter was altered by replacing it with some other letter that had not appeared in the series. The 24 two-letter foils were constructed by replacing one of the two letters with a letter that had not appeared in the sequence. Twelve foils were constructed by replacing the first letter and 12 were constructed by replacing the second letter. The foils for the three-letter displays were constructed in a similar manner. Each possible display was altered by replacing either the first, second, or third letter with a letter that had not appeared in the series. Of the 21 three-letter foils, 7 had the first letter altered, 7 had the second letter altered, and 7 had the third letter altered.

The subjects were 24 introductory psychology students who participated as part of a course option. When they arrived for the experiment, the nature of the task was described to them, and that was followed by their memorizing the base sequence. Before the test stage began, it was described in detail, and they were informed that while their reaction times were being measured it was vitally important that they make no errors. In the event subjects did make an error, the fact was recorded, but that item was presented again later in the series of displays and only the reaction time for the correct response was recorded. If a subject began to make a number of errors during the test, he was asked to study the sequence again, and he was again told of the importance of accuracy. In this way, errors were kept below $5 \%$.

\section{RESULTS}

The mean latency for each type of display (i.e., one, two, or three letters) for each serial position is given in Table 1, along with the error frequencies. For the single-letter displays, only the latencies for the "yes" responses are presented, because serial position is not meaningful for the foils. The mean latency for the "no" responses was $1.576 \mathrm{msec}$, with a standard error of .03 .

An analysis of the latencies for single letters indicated a more or less linear increase in reaction time from the first position to the last $[\mathrm{F}(8,184)=3.61$, $\mathrm{p}<.001]$. The linear component of the function was reliable $[F(1,184)=24.49, p<.001]$, but the residual was not $(F<1.00)$, and that would suggest that when subjects are asked to indicate whether a single letter appears within an organized sequence in memory, they engage in serial scan from the first position to the last. In addition, nothing unusual seemed to have occurred at the interchunk transitions (i.e., between Items 3 and 4 and between Items 6 and 7). The mean item-to-item intrachunk latency increase was $56 \mathrm{msec}$, while the mean interchunk increase was $51 \mathrm{msec}$. Therefore, the subjects did not appear to be particularly delayed as they made 
Table 1

Mean Latency in Milliseconds for Each Serial Position for Each Condition

\begin{tabular}{|c|c|c|c|c|c|c|c|c|c|c|c|c|}
\hline \multicolumn{3}{|c|}{ Letter Display } & \multicolumn{5}{|c|}{ Bigram Display } & \multicolumn{5}{|c|}{ Trigram Display } \\
\hline Serial & & & \multirow{2}{*}{$\begin{array}{l}\text { Serial } \\
\text { Position }\end{array}$} & \multicolumn{4}{|c|}{ Latency } & \multirow{2}{*}{$\begin{array}{c}\text { Serial } \\
\text { Position }\end{array}$} & \multicolumn{4}{|c|}{ Latency } \\
\hline Position & \multicolumn{2}{|c|}{ Latency } & & \multicolumn{2}{|c|}{ Yes } & \multicolumn{2}{|c|}{ No } & & \multicolumn{2}{|c|}{ Yes } & \multicolumn{2}{|c|}{ No } \\
\hline 1 & 1172 & ( 1$)$ & 12 & 1466 & ( 2$)$ & 1859 & $(0)$ & 123 & 1547 & (2) & 1849 & (3) \\
\hline 2 & 1391 & ( 0$)$ & 23 & 1509 & ( 1$)$ & 1795 & (2) & 234 & 2309 & (17) & 1931 & (1) \\
\hline 3 & 1384 & ( 2) & 34 & 1953 & ( 8$)$ & 1886 & (3) & 345 & 2367 & (11) & 2100 & (4) \\
\hline 4 & 1461 & (4) & 45 & 1762 & (3) & 1877 & (1) & 456 & 1830 & ( 2$)$ & 2044 & (2) \\
\hline 5 & 1478 & ( 2) & 56 & 1633 & $(7)$ & 1608 & (0) & 567 & 2420 & (10) & 2340 & (2) \\
\hline 6 & 1483 & (3) & 67 & 2061 & (10) & 1760 & (1) & 678 & 2533 & (10) & 1833 & (2) \\
\hline 7 & 1507 & ( 1$)$ & 78 & 1609 & ( 5$)$ & 1840 & (1) & 789 & 1621 & (4) & 1902 & (3) \\
\hline 8 & 1604 & $(7)$ & 89 & 1840 & (3) & 1972 & (2) & & & & & \\
\hline 9 & 1607 & (10) & & & & & & & & & & \\
\hline
\end{tabular}

Note-The values within parentheses are the error frequencies for each condition, although only correct responses were used to determine the latencies.

the interchunk transition. Finally, the overall error rates for the "yes" and "no" responses for the single-letter displays were .05 and .02 , respectively.

The results from the two-letter and three-letter displays were somewhat different from the single-letter displays, in that they showed much greater irregularity, and the increase from the first to the last serial positions was somewhat less clear, although there was such an increase. One prominent feature of these results, however, is that the displays that contain letters from different chunks had a much longer latency than displays that only had items from the same chunk. For the two-letter displays, the between-chunks transitions are represented by displays consisting of Items $3-4$ and Items 6-7. For three-letter displays, the between-chunks transitions occurred in displays consisting of 2-3-4, 3-4-5, 5-6-7, and 6-7-8. It would appear that a subject's performance is slowed any time he has to match a display with the contents of two different chunks, although the effect is clearer for the "yes" responses than the "no" responses.

The chunk-boundary effects are somewhat more apparent in the presentation in Table 2. Each subject was scored for his mean latency in responding to displays that did not contain an interchunk transition (within-chunks displays) and his mean latency to displays that did contain a chunk transition (betweenchunks displays). In addition, the error rates are included for each condition. The overall error rate was .048 and, with conditions as the sampling unit, the correlation between errors and latency was .26 . That would suggest that the latency effects were not attributable to a speed-accuracy tradeoff.

An analysis of the latency results indicated that the latency for three-letter displays was longer than for the two-letter displays $[F(1,23)=14.28, \mathrm{p}<.01]$ and the mean reaction time to displays containing a betweenchunks transition was longer than for displays that did not contain such a transition $[\mathrm{F}(1,23)=20.31$, $\mathrm{p}<.001]$. In addition, there was a significant Type of Response (yes vs. no) by Type of Transition interaction $[F(1,23)=38.20, p<.001]$ and a significant Length of Display by Type of Transition interaction $[F(1,23)=10.26, p<.01]$. None of the other effects were reliable (all Fs $<1.00$ ).

The interactions indicate that while having a betweenchunks transition in the display had a retarding effect on identification time, it was much greater for the positive responses than the negative responses, and the effect increased with the longer displays. A separate analysis of the "no" responses indicated that while there was an effect of length $[F(1,23)=9.77, p<.01]$, the influence of type of transition was not significant $[F(1,23)<1.00]$. That is, if the display contained a letter that did not appear in the sequence, the subjects' rejection of the display was influenced by how many letters it contained, but it was not slowed by having it contain a between-chunks transition.

A similar analysis of the "yes" responses indicated a length effect $[F(1,23)=10.90, p<.01]$, a very marked effect of type of transition $[F(1,23)=37.98$, $\mathrm{p}<.001]$, and a Length by Type of Transition

Table 2

Mean Reaction Times for the Two Types of Transitions

\begin{tabular}{|c|c|c|c|c|c|}
\hline \multirow{2}{*}{\multicolumn{2}{|c|}{$\begin{array}{l}\text { Length of } \\
\text { Sequence }\end{array}$}} & \multicolumn{4}{|c|}{ Type of Transition } \\
\hline & & $\begin{array}{l}\text { Wit } \\
\text { Chu }\end{array}$ & $\begin{array}{l}\text { nin } \\
\text { nks }\end{array}$ & $\begin{array}{l}\text { Between } \\
\text { Chunks }\end{array}$ & Both \\
\hline Two Letters & $\begin{array}{l}\text { Yes } \\
\text { No } \\
\text { Both }\end{array}$ & $\begin{array}{l}1.649 \\
1.849 \\
1.689\end{array}$ & $\begin{array}{l}(.05) \\
(.01)\end{array}$ & $\begin{array}{ll}2.006 & (.12) \\
1.823 & (.03) \\
1.915 & \end{array}$ & $\begin{array}{l}1.828 \\
1.886\end{array}$ \\
\hline Three Letters & $\begin{array}{l}\text { Yes } \\
\text { No } \\
\text { Both }\end{array}$ & $\begin{array}{l}1.666 \\
1.931 \\
1.799\end{array}$ & $\begin{array}{l}(.04) \\
(.04)\end{array}$ & $\begin{array}{ll}2.412 & (.17) \\
2.087 & (.03) \\
2.250 & \end{array}$ & $\begin{array}{l}2.039 \\
2.009\end{array}$ \\
\hline Both Lengths & $\begin{array}{l}\text { Yes } \\
\text { No } \\
\text { Both }\end{array}$ & $\begin{array}{l}1.657 \\
1.890 \\
1.774\end{array}$ & & $\begin{array}{l}2.209 \\
1.955 \\
2.082\end{array}$ & $\begin{array}{l}1.933 \\
1.923\end{array}$ \\
\hline
\end{tabular}

Note-The values within parentheses are the error rates for each condition, although only correct responses were used to determine the latencies. 
interaction $[F(1,23)=13.95, p<.01]$. Regarding the differential effect of length on the between-chunks and within-chunks transitions for the "yes" responses, there was an effect of length on the between-chunks transitions $[F(1,23)=23.80, p<.001]$, but no such effect for the within-chunks transitions $(F<1.00)$.

In summary, there was an effect of display size for the "no" responses and for between-chunks "yes" responses, but not for within-chunks "yes" responses. In addition, a between-chunks display slowed down the "yes" responses but not the "no" responses.

\section{DISCUSSION}

In terms of the nature of the scan, the present results with the single-letter probes suggest it to be both serial and self-terminating. That is, the fact that there appeared to be a linear increase in reaction time from the first serial position to the last, with no evidence of a larger increase at the interchunk transitions than at the intrachunk transitions, suggests that subjects scanned the sequence in serial order, beginning with the first item, and then terminated their scan and initiated the response the instant the probe item was first detected.

Although a more typical outcome in a memory scanning task is evidence for an exhaustive scan (i.e., in this case, no serial position effect), the present task does seem to fit those special conditions under which Sternberg (1969) has suggested a self-terminating search might be expected. In particular, the overall task was rather complex, because the single-letter and multiletter probes were randomly intermixed during the presentation. In the event that a multiletter probe consisted of items from more than one chunk, it would be necessary for the subject to have some idea of the position of each comparison in order to know if he should attempt to make a second comparison between the remaining items in the probe and the contents of the next chunk. That may have resulted in a general strategy for the subjects to keep track of the position of each comparison, even for single-letter probes, and Sternberg (1969) has suggested that such positionrelevant tasks should yield evidence of a self-terminating search. In addition, a second characteristic of tasks that appear to involve a self-terminating search is that they have unusually long latencies, and those obtained in the present experiment were two to three times larger than those usually obtained. Overall, then, the results would suggest a serial and self-terminating search, and the conditions of the experiment seem to be exactly those under which such an effect would be expected.

A result somewhat more germane to the present issue, however, is the complete absence of any evidence of unusually long latencies at the chunk boundaries. In fact, the mean interchunk latency was somewhat less than the mean intrachunk latency. While that effect is being taken as evidence that the organization provided a mechanism for a smooth interchunk transition, it also would be obtained if the subjects had simply disregarded the efforts to get them to organize the sequence into three chunks of three letters each. The latter possibility can be ruled out, however, because of the difference in latency for the between-chunks and within-chunks probes when the probe consisted of more than one item. That is, those differential effects would be dependent upon the subjects' having used the chunking organization.

Given this evidence that the organization was used, as well as the fact that interchunk transitions were smooth in the case of a single-letter probe, a reasonable interpretation of the data is that subjects did retrieve the items from a chunk as they were scanning the items from the immediately preceding chunk. Inasmuch as the within- vs. between-chunks comparison for the multiletter displays would indicate that the items from the same chunk are available simultaneously, while the items from succeeding chunks are available successively, the smooth transition from one chunk to another for a single-letter probe would imply the immediate availability of the items from the succeeding chunk. If it had been necessary for some retrieval process to intervene at that point, but not at item-to-item transitions within a chunk, a somewhat longer latency would be expected at interchunk transitions.

\section{A General Scanning Model \\ for Organized Sequences}

The following general scanning model might be a reasonable account of these results. The basic assumption is that when a display appears, the subject retrieves the sequence from memory in a chunk-bychunk manner, and each time a chunk is retrieved, he attempts to match the presented display to that chunk or a segment of that chunk. In addition, it is assumed that the subject attempts to match the entire display as a unit to part or all of the retrieved chunk. If a match occurs, the response is "yes," but if no component of the display matches a component of the retrieved chunk, the subject retrieves another chunk, and again attempts to make a match. Again, if a match occurs, the subject responds "yes," and if a complete mismatch occurs, he retrieves another chunk and once more attempts to make a match.

If, at any time, a partial match occurs, there are two possibilities. Either the display is a foil and contains an item not belonging to the sequence, or it is a segment of the sequence but contains letters from more than one chunk. In either event, the subject must then make a letter-by-letter match between the letters in the display and the letters in the retrieved chunk to determine which ones do match. In order to test the letters that do not match, he must then compare them with the contents of the next chunk. Given such a matching 
procedure, it is clear that if a complete match did occur for a between-chunks display, the subject could have responded "yes" only after he had individually matched every letter in the display, while, in the case of a mismatch, he could have responded "no" when he first encountered any mismatching letter.

In general, then, the explanation assumes that at the time of the display, the sequence is retrieved in a chunk-by-chunk manner, and subjects attempt to match the contents of the display to the contents of the retrieved chunks. In the event of a mismatch, the subjects either attempt to make a match with the contents of the next chunk or, in the case of the partial match, they make a letter-by-letter comparison between the display and the contents of memory.

This general scanning model seems to be an adequate account of the results obtained in this task with both the single-letter and the multiletter probes, with the exception that two additional assumptions are needed to handle the apparent influence of order on the subjects' performance. For both tasks there appeared to be an increase in reaction time as the position of the probe item moved from the beginning of the sequence to the end, although the effect was both slight and a bit ambiguous for the multiletter probes. However, these results would seem to imply that not only was the processing serial, but it also seemed to occur in the direction of the first item to the last. The second assumption is the point noted above that the scan and retrieval processes included in the model can occur in parallel, and this point also seems to be required by the data.

The way this construction of the processing would account for the single-probe task is quite straightforward in that it implies a first-to-last scan, with the items in each chunk being available by the time the scan reaches them. For the multiletter probes, the application of the model is somewhat more complex, but equally clear. For example, in that it assumes that the matching is done on a chunk-by-chunk basis, the longer latencies for the between-chunks displays would be expected, because subjects must compare the contents of the display to two chunks rather than just one. In addition, it assumes that when subjects compare displays to the contents of a remembered chunk, they do so by treating the display as a single unit. Given that, if a match occurs there should be no effect of length of display on identification time. On the other hand, the model predicts a letter-by-letter matching whenever a mismatch occurs, and a mismatch would occur for: (1) any withinchunks display that contained an incorrect letter, (2) a between-chunks display that contained an incorrect letter, or (3) a between-chunks display that did not contain an incorrect letter (i.e., a "yes"). In all three of these cases, significant length effects were obtained, while no such effects occurred for the "yes" responses when all of the letters came from the same chunk.
The differential pattern of latencies for the "yes" and "no" responses also can be handled by this explanation. For within-chunks displays, a "yes" response can be made on the basis of the single match, regardless of the length of the sequence, but a "no" response can occur only after a letter-by-letter match, as well as a retrieval and attempted matching of the contents of the subsequent chunk. That would result in not only predicting a length effect for the "no" responses, but predicting that their latency should be longer than for the "yes" responses. These results were obtained. For the between-chunks transitions, on the other hand, an initial mismatch will occur for both the "yes" and "no" responses which, in both cases, will entail both a letter-by-letter match and the retrieval of more than one chunk. However, in that a "yes" response can occur only after subjects have matched all the letters in the display, and a "no" response can occur at the time of the first mismatch, one would expect a longer latency for the "yes" responses than the "no" responses, and that also was obtained.

\section{CONCLUSIONS}

The major focus of the present paper has been on the role of organization in directing a memory scan and an assessment of the extent to which organization allows subjects to retrieve information from one chunk as they are scanning the information from chunks earlier in the sequence. The latter point is an important implication of a somewhat more general model of the role of organization in retrieval and recall, and has been used as the basic mechanism for explaining the ease with whch subjects can generate high-speed sequences, such as familiar telephone numbers or the alphabet.

The results seem to be generally in accord with expectations. While the information from one chunk does not appear to be immediately available to a subject as he is scanning the preceding chunk, the information does appear to be immediately available by the time the scan of the preceding chunk is complete. That would at least suggest that these two scan and retrieval processes can occur in parallel. In addition, the scan itself seemed to be serial and self-terminating, although it did appear that the letters within multiletter probes were initially compared to the contents of a chunk in a parallel fashion. That is, if the letters in a probe matched the contents of a single chunk, the response was very fast and not influenced by the size of the probe, but if there was an initial mismatch, there was evidence for a subsequent letter-by-letter comparison (i.e., probe size did influence the reaction time). It also was true, however, that the evidence for a first-tolast order of scan and comparison was considerably more ambiguous for the multiletter probes than for the single-letter probes.

Overall, then there did appear to be a marked 
influence of a sequence's organization on the way subjects scanned the sequence, and the influence was evident in both the retrieval and comparison stages of the scan. Such an outcome might suggest a basic similarity between a memory scan situation and somewhat more traditional retrieval tasks which involve overt recall.

\section{REFERENCES}

Bower, G. H., \& Winzenz, D. Group structure, coding, and memory for digit series. Journal of Experimental Psychology Monograph, 1969, 80(May, Part 2), 1-17.

CRaIN, R. D., \& DE Rosa, D. V. Retrieval of information from multiple ensembles in short-term memory. Memory \& Cognition, 1974, 2, 255-260.

НомA, D. Organization and long-term memory search. Memory \& Cognition, 1973, 1, 369-379.

Johnson, N. F. The role of chunking and organization in the process of recall. In. G. Bower (Ed.). The psychology of learning and motivation (Vol. IV). New York: Academic Press. 1970.
Johnson, N. F. Organization and the concept of a memory code. In A. Melton \& E. Martin (Eds.), Coding processes in human memory. Washington, D.C: H. V. Winston, 1972.

Johnson, N. F., \& Migdoll, D. M. Transfer and retroaction under conditions of changed organization. Cognitive Psychology, 1971, 2, 229-237.

LASHLEY, K. S. The problem of serial order in behavior. In L. A. Jeffress (Ed.), Cerebral mechanisms in behavior. New York: Wiley, 1951

Sternberg. S. Memory scanning: Mental processes revealed by reaction-time experiments. American Scientist, 1969. 57, 421-457.

Sternberg, S. Memory scanning: New findings and current controversies. Quarterly Journal of Experimental Psychology, 1975, 27, 1-32.

\section{NOTE}

1, I am indebted to an anonymous reviewer for pointing out this fact.

(Received for publication August 16, 1977; accepted January 16, 1978.) 